\title{
Vacinação contra hepatite B e fatores associados entre profissionais da enfermagem de um hospital universitário
}

\author{
Hepatitis $B$ vaccination and associated factors among nursing personnel at a university hospital \\ Vacunación contra la hepatitis $B$ y factores asociados entre profesionales de la enfermería en un \\ hospital universitario
}

Mônica Ferreira de Aguiar'; Cássio de Almeida Lima"'; Jair Almeida Carneiro "II; Andréa Maria Eleutério de Barros Lima Martins ${ }^{\prime v}$; Jaciara Aparecida Dias Santos ${ }^{v}$; Fernanda Marques da Costa ${ }^{\text {VI }}$

\begin{abstract}
RESUMO
Objetivo: identificar a cobertura vacinal contra Hepatite B dos profissionais de enfermagem de um hospital universitário e investigar os fatores associados à vacinação contra HB entre tais profissionais. Método: estudo transversal e analítico com abordagem quantitativa, conduzido entre agosto e novembro de 2013, em Montes Claros - Minas Gerais. Aplicou-se questionário abordando variáveis sociodemográficas e ocupacionais. As associações foram investigadas por meio da análise múltipla (regressão de Poisson), no Statistical Package for the Social Sciences 18.0. Projeto aprovado no Comitê de Ética em Pesquisa, Parecer Consubstanciado 2882/2011. Resultados: participaram 399 profissionais, destes $71 \%$ relataram ter tomado três doses da vacina. Houve associações entre prevalência de vacinação e idade, categoria profissional, carga horária semanal, turno de trabalho, satisfação com o trabalho e capacitação. Conclusão: a prevalência de vacinação contra hepatite b não atingiu as metas preconizadas e este associada a importantes fatores sociodemográficos e ocupacionais.
\end{abstract}

Palavras-chave: Vacinação; hepatite B; enfermagem; saúde do trabalhador.

\section{ABSTRACT}

Objective: to identify Hepatitis B vaccination coverage of nursing personnel at a university hospital and investigate the factors associated with $\mathrm{HB}$ vaccination among them. Method: this quantitative cross-sectional analytical study was conducted between August and November 2013 in Montes Claros, Minas Gerais. A questionnaire addressing sociodemographic and occupational variables was applied. Associations were investigated by multivariate analysis (Poisson regression), using the Statistical Package for the Social Sciences 18.0. The project was approved by the Research Ethics Committee (Documented Opinion 2882/2011). Results: participants were 399 nursing personnel, $71 \%$ of whom reported having taken three doses of the vaccine. Associations were found between vaccination prevalence and age, professional category, weekly workload, work shift, job satisfaction and training. Conclusion: hepatitis B vaccination prevalence did not meet recommended goals and was associated with important sociodemographic and occupational factors.

Keywords: Vaccination; hepatitis B; nursing; occupational health.

\section{RESUMEN}

Objetivo: identificar la cobertura vacunal contra la Hepatitis B de los profesionales de enfermería de un hospital universitario e investigar los factores asociados a la vacunación contra HB entre dichos profesionales. Método: estudio transversal analítico, con enfoque cuantitativo, realizado entre agosto y noviembre de 2013, en Montes Claros - Minas Gerais. Se aplicó un cuestionario sobre las variables sociodemográficas y ocupacionales. Se investigaron las asociaciones por medio del análisis múltiple (regresión de Poisson), en el Statistical Package for the Social Sciences 18.0. Proyecto aprobado por el Comité de Ética en Investigación, Dictamen consolidado 2882/2011. Resultados: participaron 399 profesionales, de éstos un $71 \%$ relató haber tomado tres dosis de la vacuna. Hubo asociaciones entre prevalencia de vacunación y edad, categoría profesional, tiempo de trabajo semanal, turno de trabajo, satisfacción con el trabajo y capacitación. Conclusión: la prevalencia de vacunación contra hepatitis B no alcanzó las metas preconizadas y está asociada a importantes factores sociodemográficos y ocupacionales. Palabras clave: Vacunación; hepatitis B; enfermería; salud laboral.

\section{INTRODUÇÃO}

A hepatite $B(\mathrm{HB})$ representa uma das patologias de maior relevância na saúde pública em todos os continentes. A infecção pelo vírus da HB (VHB) é considerada uma das principais causas de doença aguda e crônica do fígado. Sua transmissão ocorre principal- mente pelo contato com fluidos corporais, pelas vias parenteral, sexual e vertical. Ainda, através de veículos como o sangue, e pode se propagar por contato com sêmen, saliva, suor, lágrimas, leite materno e efusões patológicas $^{1-3}$.

Especialista em Vigilância e Controle de Infecções Relacionadas à Assistência à Saúde pela Universidade Estadual de Montes Claros. Montes Claros, Minas Gerais, Brasil. E-mail:mofeag@yahoo.com.br

"Mestre em Saúde, Sociedade e Ambiente pela Universidade Federal dos Vales do Jequitinhonha e Mucuri. Montes Claros, Minas Gerais, Brasil. E-mail: cassioenf2014@gmail.com "'Doutorando do Programa de Pós-Graduação em Ciências da Saúde da Universidade Estadual de Montes Claros. Professor das Faculdades Integradas Pitágoras de Montes Claros. Montes Claros, Minas Gerais, Brasil. E-mail: jairjota@yahoo.com.br

"Voutora em Epidemiologia. Professora do Programa de Pós-Graduação em Ciências da Saúde da Universidade Estadual de Montes Claros. Montes Claros, Minas Gerais, Brasil. E-mail: martins.andreamebl@gmail.com

vAcadêmica do Curso de Graduação em Medicina da Universidade Estadual de Montes Claros. Montes Claros, Minas Gerais, Brasil. E-mail: jaciarapds@gmail.com

v'Doutora em Ciências da Saúde. Professora das Faculdades Integradas Pitágoras de Montes Claros. Montes Claros, Minas Gerais, Brasil. E-mail: fernandafijf@yahoo.com.br 
Existem aproximadamente 325 milhões de portadores crônicos do VHB em diversas regiões do mundo e estima-se que mais de 500 mil morram, anualmente, vítimas dessa enfermidade ${ }^{1,4}$. No Brasil, o Ministério da Saúde estima que $15 \%$ da população já foi exposta ao VHB, e que $1 \%$ sofra de HB crônica². No estado de Minas Gerais (MG) - Brasil, foram notificados 6.525 casos entre 2010-2015, destacando-se maior frequência na faixa etária de 10 a 19 anos e na região Norte, além de grande desconhecimento da população a respeito da doença ${ }^{5}$.

Contudo, no Brasil o acesso à vacina contra a doença é público e sua distribuição ocorre sem custos para os usuários do Sistema Único de Saúde (SUS). No país, houve uma importante redução nos níveis de endemia, como resultado de estratégias nacionais de controle, sobretudo a vacinação de lactentes, adolescentes e adultos com idade igual ou superior a 49 anos. Informa-se que os trabalhadores da área da saúde são vacinados desde 1993 e a cobertura de séries completas variaram entre 53\% e 76\% nos anos de 2005, 2006 e $2010^{6}$.

Os trabalhadores da saúde, ao realizarem suas atividades, se expõem, principalmente, ao contato com material biológico. Isso pode predispor ao risco de adquirir infecções veiculadas pelo sangue, dentre elas a infecção pelo VHB, uma vez que as suas atividades requerem contato frequente com o paciente, principalmente a manipulação de materiais perfurocortantes e de fluidos corporais ${ }^{7}$. Nos hospitais, locais insalubres, os trabalhadores da área da saúde são expostos a riscos ocupacionais ${ }^{8}$. Dentre esses trabalhadores, os profissionais da enfermagem, durante a assistência prestada ao paciente, são expostos a diversos riscos: físicos, químicos, mecânicos, biológicos, ergonômicos, que podem comprometer a saúde e propiciar a ocorrência de acidentes de trabalho ${ }^{4}$.

A situação de risco dos trabalhadores da enfermagem merece atenção especial quanto às medidas de prevenção contra o VHB, sendo a vacinação a melhor forma de proteção e uma das intervenções mais relevante de saúde pública. A vacinação dos trabalhadores da saúde pode diminuir a incidência de infecção pelo VHB em até $95 \%{ }^{2}$. A Norma Regulamentadora 32 (NR 32) estabelece diretrizes básicas para implementar medidas de proteção à segurança e à saúde dos trabalhadores dos serviços de saúde, bem como daqueles que exercem atividades de promoção e assistência à saúde. São preconizados o uso de EPI, a higienização das mãos, a vacinação contra HB, entre outras disposições ${ }^{9}$.

Face à essa realidade, informações epidemiológicas atualizadas são essenciais e necessárias, o que evidencia a relevância de pesquisas que investiguem a situação vacinal dos profissionais. Na literatura ${ }^{3,6,8,10-14}$, recomenda-se fortemente o estudo da prevalência e dos fatores associados à vacinação contra HB entre profissionais da enfermagem, caracterizando aqueles que não vacinaram, a fim de contribuir para o direcionamento de ações que visem diminuir a incidência dessa grave doença. É necessário melhor elucidar os fatores determinantes para a vacinação, para que se aprofunde o conhecimento sobre o problema e sejam feitos investimentos na saúde do trabalhador, em conformidade com as características daqueles profissionais que não vacinaram ${ }^{3,6,8,10-14}$.

Tais estudos ainda podem contribuir para a prevenção e o controle de riscos nos ambientes de trabalho, para o planejamento de ações e estratégias efetivas de conscientização sobre as formas de controle e prevenção das infecções, assim como para a promoção da saúde do trabalhador ${ }^{3,13,14}$. E, considerando a alta vulnerabilidade dos profissionais da enfermagem à $\mathrm{HB}$, acredita-se que o presente trabalho pode fomentar subsídios à vigilância epidemiológica referente à $\mathrm{HB}$ e à saúde do trabalhador. Este estudo teve por objetivos: identificar a cobertura vacinal contra $\mathrm{HB}$ dos profissionais de enfermagem de um hospital universitário e investigar os fatores associados à vacinação contra HB entre tais profissionais.

\section{REVISÃo DE LITERATURA}

Nas últimas décadas, inúmeras iniciativas da sociedade brasileira vêm procurando consolidar e implantar avanços na política pública de atenção integral à saúde do trabalhador. Sobretudo na década de 1970, quando o movimento da saúde do trabalhador no Brasil se fortaleceu, norteado pela defesa do direito ao trabalho digno e saudável, com a participação dos profissionais nas decisões sobre a gestão dos processos produtivos e na busca da garantia da atenção integral à saúde. Contudo, pode-se destacar que a política de Estado, constituída naquele período, prevalece até os dias atuais, sem alterações substanciais ${ }^{10,11}$.

Nesse sentido, faz-se necessário o investimento dos gestores em recursos humanos com vistas ao seu estímulo e o desenvolvimento profissional, sendo necessária a reorganização das normas organizacionais e a mobilização dos trabalhadores. Assim, estes podem alcançar melhorias de suas práticas em saúde à luz do autocuidado, além de poderem expressar suas potencialidades e dificuldades no trabalho ${ }^{15,16}$. Todavia, apesar dos esforços no sentido de elaboração de políticas de proteção à saúde do trabalhador, cuja expressão mais atual se dá com a NR 32, ainda há falhas no que concerne à vigilância aos trabalhadores no contexto da vacinação e dos acidentes com exposição a materiais biológicos ${ }^{7,17}$.

É necessário que a vigilância em saúde do trabalhador seja um elemento estruturado no Sistema Único de Saúde (SUS). Para tanto, é imprescindível que suas ações estejam inseridas no cotidiano das equipes de saúde, como elemento obrigatório para a construção da integralidade na atenção à saúde. A vacinação completa, somada à comprovação sorológica dos profissionais da saúde, é uma condição imprescindível na prevenção da transmissão ocupacional da HB, indicando a possibilidade de se ampliar as intervenções de prevenção e de proteção ${ }^{18}$.

Entre as diversas práticas de proteção à saúde do trabalhador, destaca-se a vacinação, enquanto medida prática e eficiente de prevenção para determinadas doen- 
ças presentes no ambiente hospitalar. A imunização é um meio de proteção contra diversas enfermidades graves, às quais os trabalhadores de enfermagem estão em contínua exposição. Reitera-se que é uma ação que deve preceder os exames ocupacionais, sendo uma intervenção específica para os fatores de risco biológico imunopreveníveis ${ }^{1,10}$.

Atualmente, o VHB é considerado o vírus com maior possibilidade de transmissão ocupacional, mas já existe proteção garantida pelo imunizante. Contudo, para ter a garantia de proteção, é necessário que os profissionais, além de imunizados, realizem o exame sorológico para detecção de anticorpos circulantes que conferem proteção para a HB, já que nem todos os indivíduos vacinados se soroconvertem ${ }^{18}$. A HB é uma doença ocupacional relevante no ambiente de saúde e os profissionais da enfermagem estão susceptíveis a adquiri-la, se medidas de precaução, tanto coletivas quanto individuais, não forem implementadas. Então, recomenda-se a ampliação de estratégias de educação em serviço e permanente, bem como o desenvolvimento de pesquisas sobre saúde do trabalhador ${ }^{14}$.

Apesar da universalização da imunização, muitos profissionais estão com esquemas aquém dos mínimos necessários para garantia de proteção contra a HB ${ }^{18}$. O enfrentamento das barreiras relacionadas ao ato de imunizar-se ainda é uma tarefa que demanda um compromisso contínuo da área de vigilância em saúde do trabalhador, para que se assegure o caráter real de proteção a esse grupo ${ }^{17,18}$.

\section{Metodologia}

Trata-se de um estudo transversal, descritivo e analítico, realizado entre agosto e novembro de 2013 entre profissionais da enfermagem de um hospital universitário de Montes Claros, MG - Brasil.

No hospital universitário atuavam 487 profissionais da enfermagem, sendo 93 auxiliares de enfermagem, 296 técnicos de enfermagem e 98 enfermeiros. 0 número de sujeitos investigados foi definido por cálculo amostral, que utilizou os seguintes parâmetros: grau de confiança de $95 \%$, proporção de $50 \%$ e erro de $2 \%$. Nesse sentido, a amostra estimada foi de 406 trabalhadores. Foram incluídos na investigação os profissionais que aceitaram responder o questionário proposto e que estavam presentes no local de trabalho, sendo realizadas até três tentativas de aplicação do questionário em momentos diferentes. Os critérios de exclusão foram: estar em férias, em atestado ou licença médica.

Os dados foram coletados por meio de questionário autoaplicável. A variável dependente foi investigada mediante o autorrelato de vacinação, sendo considerados vacinados os trabalhadores que tomaram três doses da vacina contra $\mathrm{HB}$, e não vacinados aqueles que tomaram uma, duas ou nenhuma dose. As variáveis independentes foram assim agrupadas:

-Aspectos sociodemográficos: idade, sexo, estado civil (com companheiro e sem companheiro), escolaridade, renda mensal. Exceto sexo e estado civil, as demais variáveis foram categorizadas tendo a mediana como ponto de corte.

-História ocupacional: cargo na instituição hospitalar, categorizado em três níveis: superior (enfermeiro), médio (técnicos de enfermagem) e fundamental (auxiliar de enfermagem); tempo de trabalho na profissão; tempo de trabalho na instituição hospitalar; regime de trabalho (dicotomizado em efetivo ou contratado); carga horária semanal (até 39 horas e 40 horas ou mais); turno de trabalho (diurno, noturno, diurno e noturno); autorrelato de contato com material perfurocortante. As variáveis quantitativas tempo de trabalho na profissão, tempo de trabalho no hospital e carga horária semanal foram categorizadas considerando a mediana como ponto de corte.

-Satisfação com o trabalho: satisfeito (considerando as categorias muito satisfeito e satisfeito) ou insatisfeito (considerando as categorias moderadamente satisfeito e insatisfeito). Também foi investigado se o trabalhador havia sido convidado a se vacinar contra a HB depois que ingressou no hospital e se participou de curso de capacitação e/ou discussão a respeito de saúde do trabalhador promovida pela instituição.

Após a coleta, construiu-se um banco de dados eletrônico, no programa Excel $^{\circledR}$. Os dados coletados foram processados em dupla entrada e, posteriormente, validados para verificar a consistência entre as duas bases. Em seguida, o referido banco foi transportado para o software Statiscal Package for the Social Sciences (SPSS), versão 18.0, para proceder às análises. Utilizaram-se frequências absolutas e percentuais para a análise descritiva. Após análise descritiva, a associação entre o autorrelato de vacinação e as variáveis independentes foi investigada por meio da análise bivariada e da regressão de Poisson multivariada (com estimador robusto), com estimativa de razões de prevalência bruta e ajustada. Foram incluídas na análise multivariada as variáveis associadas ao relato de vacinação que tiveram um valor $p \leq 0,20$ na análise bivariada. $\mathrm{O}$ modelo final foi ajustado, mantendo-se as variáveis associadas a um valor $p \leq 0,05$. Adotou-se um nível de significância de 95\%.

Os aspectos éticos da pesquisa foram considerados, conforme a Resolução no 466/2012 do Conselho Nacional de Saúde. O projeto de pesquisa foi aprovado pelo Comitê de Ética em Pesquisa da Universidade Estadual de Montes Claros (UNIMONTES), mediante o Parecer Consubstanciado 2882. Todos os participantes leram e assinaram o Termo de Consentimento Livre e Esclarecido.

\section{RESULTADOS}

Dos 487 profissionais da enfermagem do hospital universitário, 406 foram sorteados a partir do cálculo amostral. Destes, 400(98,5\%) aceitaram responder o questionário proposto e 399(98,2\%) responderam a questão sobre a vacinação contra HB. Entre os participantes, 282(71\%) relataram ter tomado três doses da vacina contra HB e $117(29 \%)$ não receberam nenhuma dose ou não completaram o esquema vacinal. Os fatores associados 
ao relato de vacinação contra HB na análise bivariada $(p \leq 0,20)$ foram: idade, escolaridade, renda mensal, cargo na instituição hospitalar, regime de trabalho, carga horária semanal, turno de trabalho, ter verificado imunização contra HB, satisfação com o trabalho, ter sido convidado a se vacinar quando ingressou no hospital e ter participado de curso de capacitação na área de saúde do trabalhador com ênfase na prevenção da HB, conforme mostra a Tabela 1.

TABELA 1: Resultado da análise bivariada das variáveis dos relatos de vacinação contra HB entre profissionais da enfermagem de um hospital escola. Montes Claros, MG, Brasil, 2013. ( $\mathrm{N}=399$ ).

\begin{tabular}{|c|c|c|c|c|c|c|c|}
\hline \multirow{3}{*}{ Variáveis } & \multicolumn{4}{|c|}{ Vacinação contra HB } & \multirow{3}{*}{$\mathbf{R} \mathbf{P}^{(*)}$} & \multirow{3}{*}{$\operatorname{IC95\% (**)}$} & \multirow{3}{*}{ Valor $P$} \\
\hline & \multicolumn{2}{|c|}{ Sim } & \multicolumn{2}{|c|}{ Não } & & & \\
\hline & $\mathbf{n}$ & $\%$ & $\mathbf{n}$ & $\%$ & & & \\
\hline \multicolumn{8}{|l|}{ Idade } \\
\hline Até 33 anos & 146 & 76,4 & 45 & 23,6 & 1,00 & \multirow{2}{*}{$1,01-1,16$} & \multirow{2}{*}{0,01} \\
\hline 34 anos ou mais & 136 & 65,4 & 72 & 34,6 & 1,08 & & \\
\hline \multicolumn{8}{|l|}{ Sexo } \\
\hline Masculino & 81 & 73,6 & 29 & 26,4 & 1,00 & \multirow{2}{*}{$0,95-1,11$} & \multirow{2}{*}{0,42} \\
\hline Feminino & 201 & 69,6 & 88 & 30,4 & 1,03 & & \\
\hline \multicolumn{8}{|l|}{ Estado civil } \\
\hline Com companheiro & 158 & 70,2 & 67 & 29,8 & 1,00 & \multirow{2}{*}{ 0,92-1,06 } & \multirow{2}{*}{0,82} \\
\hline Sem companheiro & 124 & 71,3 & 50 & 28,7 & 0,99 & & \\
\hline \multicolumn{8}{|l|}{ Escolaridade em anos de estudo } \\
\hline Mais de 14 anos de estudo & 133 & 76,4 & 41 & 23,6 & 1,00 & \multirow{2}{*}{ 1,01-1,16 } & \multirow{2}{*}{0,02} \\
\hline Até 14 anos de estudo & 149 & 66,2 & 76 & 33,8 & 1,08 & & \\
\hline \multicolumn{8}{|l|}{ Renda média mensal } \\
\hline Acima de 2 Salários Mínimos & 170 & 76,9 & 51 & 23,1 & 1,00 & & \\
\hline Até 2 Salários Mínimos & 112 & 62,9 & 66 & 37,1 & 1,11 & 1,04-1,19 & 0,00 \\
\hline Cargo no hospital & & & & & & & \\
\hline Enfermeiros & 72 & 86,7 & 11 & 13,3 & 1,00 & & \\
\hline Técnico de enfermagem & 204 & 67,1 & 100 & 32,9 & 1,32 & $1,08-1,61$ & 0,00 \\
\hline Auxiliar de enfermagem & 6 & 50,0 & 6 & 50,0 & 1,17 & $1,08-1,26$ & 0,00 \\
\hline Tempo de profissão & & & & & & & \\
\hline Até 8 anos & 153 & 71,8 & 60 & 28,2 & 1,00 & & \\
\hline Mais de 8 anos & 128 & 69,2 & 57 & 30,8 & 1,02 & 0,95-1,09 & 0,56 \\
\hline Tempo de trabalho no hospita & & & & & & & \\
\hline Até 4 anos & 147 & 68,1 & 69 & 31,9 & 1,00 & & \\
\hline Mais de 4 anos & 135 & 73,8 & 48 & 26,2 & 0,95 & U,89-1,U2 & 0,21 \\
\hline Regime de trabalho & & & & & & & \\
\hline Efetivo & 276 & 70,2 & 117 & 29,8 & 1,00 & $074-079$ & 011 \\
\hline Contratado & 6 & 100 & 0 & 0 & 0,77 & $0,14-0,19$ & $0, \perp 1$ \\
\hline Carga horária semanal & & & & & & & \\
\hline Até 39 horas & 178 & 65,0 & 96 & 35,0 & 1,00 & $080-092$ & 0,00 \\
\hline 40 horas ou mais & 104 & 83,2 & 21 & 16,8 & 0,86 & & \\
\hline Turno de trabalho & & & & & & & \\
\hline Diurno & 139 & 67,1 & 68 & 32,9 & 1,00 & & \\
\hline Noturno & 77 & 67,5 & 37 & 32,5 & 0,86 & $0,79-0,94$ & \\
\hline Diurno e Noturno & 66 & 84,6 & 12 & 15,4 & 0,99 & $0,92-1,08$ & U,UI \\
\hline Fez exame de sangue para veri & & & & & & & \\
\hline imune à HB & & & & & & & \\
\hline Sim & 107 & 87,7 & 15 & 12,3 & 1,00 & & \\
\hline Não & 165 & 66,5 & 83 & 33,5 & 1,18 & 1,11-1,27 & 0,00 \\
\hline Satisfação com o seu trabalho & & & & & & & \\
\hline Satisfeito & 220 & 69,0 & 99 & 31,0 & 1,00 & & \\
\hline Insatisfeito & 57 & 79,2 & 15 & 20,8 & 0,92 & $0,84-1,00$ & 0,06 \\
\hline $\begin{array}{l}\text { Convidado a se vacinar contra } \\
\text { ingressou na atenção hospitala }\end{array}$ & & & & & & & \\
\hline Sim & 236 & 72,8 & 88 & 27,2 & 1,00 & $0.99-119$ & 0.05 \\
\hline Não & 46 & 61,3 & 29 & 38,7 & 1,09 & $0,99-1,19$ & 0,US \\
\hline $\begin{array}{l}\text { Participou de capacitação na á } \\
\text { trabalhador sobre prevenção c }\end{array}$ & & & & & & & \\
\hline Sim & 128 & 80,5 & 31 & 19,5 & 1,00 & & \\
\hline Não & 154 & 64,2 & 86 & 35,8 & 1,13 & 1,06-1,21 & 0,00 \\
\hline
\end{tabular}


A análise multivariada evidenciou menor prevalência de autorrelato de vacinação entre os profissionais: mais jovens, técnicos ou auxiliares de enfermagem, que trabalham até 39 horas semanais, que atuam no turno noturno ou diurno e noturno, insatisfeitos com as atividades laborais, que participaram de capacitação referente à $\mathrm{HB}$, como demonstrado na Tabela 2. Portanto, são esses os profissionais não vacinados contra a HB.

TABELA 2: Fatores associados ao relato de vacinação contra hepatite $b$ entre profissionais da enfermagem de um hospital escola. Montes Claros, MG, Brasil, 2013. ( $N=399)$.

\begin{tabular}{|c|c|c|c|}
\hline Variáveis & $\mathbf{R} \mathbf{P}^{(*)}$ & $\begin{array}{c}\mathrm{IC} \\
95 \%^{(* *)}\end{array}$ & $\begin{array}{c}\text { Valor } \\
P\end{array}$ \\
\hline \multicolumn{4}{|l|}{ Idade } \\
\hline Até 33 anos & 1,00 & & \\
\hline 34 anos ou mais & 1,07 & $1,00-1,14$ & 0,04 \\
\hline \multicolumn{4}{|l|}{ Cargo no hospital } \\
\hline Enfermeiros & 1,00 & & \\
\hline Técnico de enfermagem & 0,64 & $0,37-1,09$ & 0,03 \\
\hline Auxiliar de enfermagem & 0,85 & $0,75-0,96$ & 0,01 \\
\hline \multicolumn{4}{|l|}{ Carga horária semanal } \\
\hline Até 39 horas & 1,00 & & \\
\hline 40 horas ou mais & 1,17 & $1,03-1,33$ & 0,01 \\
\hline \multicolumn{4}{|l|}{ Turno de trabalho } \\
\hline Diurno & 1,00 & & \\
\hline Noturno & 0,96 & $0,83-0,98$ & 0,04 \\
\hline Diurno e Noturno & 0,91 & $0,88-1,04$ & 0,37 \\
\hline \multicolumn{4}{|l|}{$\begin{array}{l}\text { Satisfação com o seu } \\
\text { trabalho }\end{array}$} \\
\hline Boa & 1,00 & & \\
\hline Ruim & 0,92 & $0,85-0,98$ & 0,04 \\
\hline \multicolumn{4}{|l|}{$\begin{array}{l}\text { Participou de capacitação } \\
\text { na área de saúde do } \\
\text { trabalhador sobre } \\
\text { prevenção da hepatite B }\end{array}$} \\
\hline Sim & 1,00 & & \\
\hline Não & 1,12 & $1,05-1,20$ & 0,00 \\
\hline
\end{tabular}

Nota: $\left({ }^{*}\right)$ RP: Razão de prevalência / $\left.{ }^{* *}\right)$ IC: Intervalo de confiança.

\section{DISCUSSÃo}

Este trabalho permitiu o conhecimento da prevalência e os fatores relacionados ao relato de vacinação contra HB entre os profissionais da enfermagem de um hospital universitário, bem como permitiu evidenciar as características associadas à menor prevalência desse relato. Dessa forma, os profissionais mais jovens, os técnicos e auxiliares de enfermagem, aqueles que trabalham até 39 horas semanais, que atuam no turno noturno ou diurno e noturno, se encontram insatisfeitos com o trabalho e participaram de capacitação referente à HB são os principais não vacinados contra a HB.

Entre os participantes da pesquisa, 282(71\%) relataram ter tomado as três doses da vacina contra HB, enquanto $117(29 \%)$ não receberam nenhuma dose ou não completaram o esquema vacinal. Já em outros hospitais universitários, observaram-se as prevalências de vacinação de $73,9 \%^{3}, 64,2 \%^{8}$ e $53,2 \%^{19}$.

Então, o hospital não alcançou as metas almejadas. Apresenta-se uma realidade inquietante, uma vez que o artigo 168 da Lei 7.855/89 recomenda a realização de exames admissionais com a comprovação de esquema vacinal completo contra a $\mathrm{HB}^{20}$. A cobertura vacinal dos profissionais de enfermagem pode ser melhorada, pois a vacina contra a HB está disponível no sistema público e é a forma mais eficaz de se prevenir a $\mathrm{HB}$, uma vez que a resposta vacinal em adultos imunocompetentes é de 90 a $95 \%{ }^{21,22}$.

Sendo assim, tais achados requerem uma detida discussão. A ausência de informação, a falta de capacitação e a baixa escolaridade são os principais fatores apontados para justificar a não vacinação contra $\mathrm{HB}^{15}$. O baixo nível de escolaridade entre os trabalhadores da saúde pode estar relacionado ao baixo conhecimento e, consequentemente, a uma menor porcentagem de vacinação, tornando tais profissionais menos protegidos ${ }^{3}$. Os profissionais que não vacinaram podem ter desconhecimento acerca das formas de transmissão da HB; não saberem a quantidade de doses preconizadas pelo Ministério da Saúde para se obter a imunização; além de apresentarem pouco conhecimento e autocuidado a respeito de sua própria saúde, negligenciando medidas importantes, como a vacinação $0^{3,23}$. Adicionalmente, a formação do profissional de saúde ainda é voltada para o tecnicismo, persistem lacunas entre o cuidado do paciente e o autocuidado do cuidador, dificultando a promoção da saúde do trabalhador de saúde ${ }^{23}$.

Situações como a falta de um plano de saúde do trabalhador adequado, com protocolos que direcionem e monitorem a realização do esquema completo contra $H B$, podem refletir em risco ocupacional importante, considerando que a chance de infecção pelo VHB para os profissionais não imunes é muito alta. Estima-se que trabalhadores da rede hospitalar que se acidentam com percutâneos contaminados possuem maior risco de se contaminar com o VHB (6\% a 30\%), visto que os índices de contaminação com o vírus da hepatite $C(0,5 \%$ a $2 \%)$ e com o vírus HIV/Aids (0,3\%) são menores, reiterando assim, a necessidade de $100 \%$ dos trabalhadores de saúde serem vacinados e monitorados quanto à imunização contra a $\mathrm{HB}^{23,24}$. Dessa forma, destaca-se o papel do Núcleo de Vigilância Epidemiológica existente no cenário desta investigação, o qual deve estar atento à situação vacinal dos profissionais da instituição, ao monitoramento da HB e à implementação de medidas preventivas.

Ao contrário do esperado, verificou-se uma menor prevalência de relato de vacinação contra HB entre os profissionais com menos de 34 anos de idade e que participaram de capacitação na área de saúde do trabalhador. Portanto, os profissionais de enfermagem 
mais jovens devem ser o alvo principal das ações de prevenção e controle a HB. É necessário ampliar os investimentos em treinamentos e campanhas de vacinação contra a HB, enfocando também a importância do conhecimento e da sensibilização sobre a prevenção da doença. Torna-se essencial fortalecer a educação continuada e permanente, enfatizando essa vacinação, a prevenção dos acidentes de trabalho, atenção na realização dos procedimentos, bem como o cumprimento das normas de biossegurança ${ }^{8}$.

Os profissionais da enfermagem com escolaridade de nível superior apresentaram maior prevalência de relato de vacinação, se comparados aos de níveis técnico e auxiliar, o que pode ser explicado pelo fato de possuírem

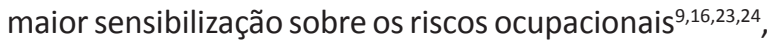
maior conhecimento sobre saúde ocupacional $\left.\right|^{3,13,15,23}$ ou porque se vacinaram durante a graduação ${ }^{3,15}$. Em outros estudos, verificou-se situação semelhante ${ }^{3,13,14,19,25-28}$, com maior frequência de vacinação entre profissionais com melhor escolaridade.

A recomendação da necessidade de se vacinar com esquema completo contra a HB já é realidade em algumas instituições de ensino superior. Isso pode ser um dos motivos que contribuíram para maior prevalência de vacinação entre trabalhadores com essa formação ${ }^{29}$. Entretanto, em estudo que investigou o conhecimento de estudantes de enfermagem acerca da vacinação contra HB, constatou-se que o conhecimento desses estudantes era parcial e variava entre as universidades. Portanto, sugere-se a determinação de um protocolo específico e claro a ser seguido por todas as instituições de ensino superior com formação em enfermagem ${ }^{30}$.

A prevalência de relato de vacinação contra HB foi menor entre os profissionais da enfermagem que trabalham até 39 horas semanais, entre os que realizam plantões no turno noturno ou diurno/noturno, e entre os que relataram estarem insatisfeitos com o trabalho. Provavelmente, os profissionais que atuam em plantões noturnos sofrem com a sobrecarga de trabalho, estão insatisfeitos com a atividade realizada e acabam por postergar o autocuidado com a sua saúde ${ }^{23}$. Resultados semelhantes foram encontrados em unidades de saúde localizadas na região norte do estado do Ceará, em que os membros da equipe de enfermagem que trabalham entre 30 a 88 horas semanais também cuidam menos da própria saúde se comparados àqueles que trabalhavam com menor carga horária semanal ${ }^{31}$.

A sobrecarga de trabalho associada ao trabalho em turnos, principalmente no turno da noite, pode interferir na qualidade de vida desses indivíduos. Tal realidade pode deteriorar a saúde do trabalhador, com o aumento de acidentes, risco de doença, exposições perigosas e menor preocupação com a segurança e saúde ocupacional, assim como menor vacinação contra $\mathrm{HB}^{19}$. Verificou-se neste estudo que, apesar de os trabalhadores investigados possuírem vínculo estável com a instituição, a prática da baixa remuneração está presente e pode interferir negativamente na satisfação com o trabalho e consequentemente na qualidade de vida e no autocuidado, reduzindo também a adesão à vacina contra a HB.

É necessário e urgente que o profissional de enfermagem reconheça sua situação de vulnerabilidade e assuma uma postura preventiva no que diz respeito à biossegurança e à sua própria saúde. Também é importante que esse profissional esteja atento às questões relativas à prevenção de doenças relacionadas ao trabalho, pois é notório que, nesta profissão, os riscos ocupacionais estão presentes, assim como a precarização das condições de trabalho ${ }^{32,33}$.

Como propostas concretas, esta investigação evidencia a necessidade de protocolos de promoção de saúde do trabalhador, com medidas gerais que incluem: esquema de vacinação completo, momentos para a realização da verificação pós-imunização, momentos para redução do estresse. Devem ser implantados programas de estímulo à atividade física e adoção de um estilo de vida saudável, instigando o trabalhador a incorporar medidas de autocuidado à saúde que poderá ser uma forma de influenciar positivamente a satisfação com o trabalho, que esteve tão prejudicada entre os profissionais investigados.

Adicionalmente, há necessidade de estímulo ao trabalhador, com planos de carreira, programas que evitem as perdas salariais ao longo do tempo, permitindo que se mantenha satisfeito no trabalho. Ressalta-se que a implementação desses protocolos contribuirá para atender o que foi preconizado pelo Ministério da Saúde por meio da Política Nacional de Saúde do Trabalhador, em 2011, quando foram publicadas as "Diretrizes da Política Nacional de Promoção de Saúde do Trabalhador do Sistema Único de Saúde", pois reconhecem que a qualidade no trabalho e a promoção de saúde estão associadas à desprecarização do trabalho e ao estabelecimento de um plano de saúde do trabaIhador concreto ${ }^{34}$.

\section{CONCLUSÃo}

A prevalência de vacinação contra a HB entre os profissionais da enfermagem do hospital universitário ainda não atingiu as metas preconizadas para a garantia da saúde desses trabalhadores, no que diz respeito à infecção pelo VHB. Os resultados evidenciaram que os profissionais mais jovens, os técnicos e auxiliares de enfermagem, aqueles que trabalham até 39 horas semanais, que atuam no turno noturno ou diurno e noturno, se encontram insatisfeitos com o trabalho e participaram de capacitação referente à HB são os principais não vacinados contra a HB.

Recomenda-se que todos os trabalhadores devem comprovar a vacinação no momento da admissão em 
serviços de saúde e que a instituição deve estar atenta à situação vacinal e de saúde ocupacional de seus profissionais. Isso merece especial destaque no cenário deste trabalho, pois se trata de um hospital de ensino que deve ser um serviço modelo para os graduandos, os quais, em sua formação, devem ter evidenciada a prevenção por meio de uma ação eficaz na quebra da cadeia epidemiológica das doenças transmissíveis: a vacinação. Então, reitera-se a necessidade de mais informações sobre a importância da vacina contra HB e da verificação da imunização pós-vacinação.

Este trabalho apresenta limitações, pois se baseou em dados autorrelatados. Logo, seus resultados podem ser superestimados, pois leva em conta o relato dos trabalhadores, que podem optar por respostas consideradas corretas, mesmo que elas não representem a realidade de sua prática. Para minimizar esse viés, os entrevistadores foram treinados, esclarecendo aos participantes sobre a confidencialidade das informações. Todavia, informações autorreferidas são comumente utilizadas em estudos epidemiológicos. Pesquisas longitudinais devem ser realizadas, a fim de esclarecer os fatores associados à não vacinação e estabelecer uma relação causal.

\section{REFERÊNCIAS}

1. Fraguás SA, Silvino ZR, Flach DMAM, Reis IR, Andrades M. Immunization against Hepatitis $B$ : a matter of occupational health nursing. Rev Pesq Cuid Fundam Online [periódico na internet]. 2013 [citado em 22 jan 2014]; 5(1): 3150-8. Disponível em: http:// www.seer.unirio.br/index.php/cuidadofundamental/article/ view/1865/pdf_671

2. Ministério da Saúde (Br). Secretaria de Vigilância em Saúde. Departamento de Vigilância Epidemiológica. Hepatites virais: o Brasil está atento. Brasília (DF): Ministério da Saúde; 2008.

3. Soares DM, Lima CA, Costa FM, Carneiro JA. Nursing: reality of immunization against Hepatitis $B$ in a hospital of the north of Minas Gerais. Esc Anna Nery. 2015; 19(4):692-701.

4. Simão SAF, Soares CRG, Souza V, Borges RAA, Cortez EA. Acidentes de trabalho com material perfurocortante envolvendo profissionais de enfermagem de unidade de emergência hospitalar. Rev enferm UERJ. 2010; 18(3):400-4.

5. Minas Gerais. Secretaria de Estado de Saúde de Minas Gerais. Subsecretaria de Vigilância e Proteção à Saúde. Superintendência de Vigilância Epidemiológica, Ambiental e Saúde do Trabalhador. Diretoria de vigilância epidemiológica. Alerta epidemiológico Junho amarelo, 2017. Belo Horizonte (MG): Secretaria de Estado de Saúde de Minas Gerais; 2017.

6. Álvarez AMR, Pérez-Vilar S, Pacis-Tirso C, Contreras M, Nathalie El O, Cuauhtémoc Ruiz-Matus, et al. Progress in vaccination towards hepatitis $B$ control and elimination in the Region of the Americas. BMC Public Health. 2017; 17:1-10.

7. Paiva MHRS, Oliveira AC. Fatores determinantes e condutas pós-acidente com material biológico entre profissionais do atendimento pré-hospitalar. Rev Bras Enferm. 2011; 64(2):268-73.

8. Ruas EFG, Santos LS, Barbosa DA, Belasco AGS, Bettencourt ARC. Acidentes Ocupacionais com Materiais Perfurocortantes em Hospitais de Montes Claros-MG. Rev Min Enferm. 2012; 16(3):437-43. 9. Ministério do Trabalho (Br). Norma Regulamentadora 32, de 11 de novembro de 2005: dispõe sobre a segurança e saúde no trabalho em serviços de saúde. Brasília (DF): Ministério do Trabalho; 2005.
10.Dias MP, Lima CJM, Nobre CS, Feijão AR. Vaccine profile of nursing professional in a hospital reference in infectious diseases in Fortaleza - Ceará. Cienc Cuid Saude. 2013; 12(3):475-82.

11. Costa D, Lacaz FAC, Filho JMJ, Vilela RAG. Saúde do trabalhador no SUS: desafios para uma política. Rev Bras Saúde Ocup. 2013; 38(127):11-30.

12. Dannetun E, Tegnell A, Torner A, Giesecke J. Coverage of hepatitis $B$ vaccination in Swedish health care workers. J Hosp Infect. 2006; 63(1):201-4.

13. Costa FM, Martins AMEBL, Santos NPE, Veloso DNP, Magalhães VS, Ferreira RC. Is vaccination against hepatitis $B$ a reality among Primary Health Care workers? Rev Latino-Am Enfermagem. 2013; 21(1):316-24.

14. Araújo TME, Costa e Silva N. Acidentes perfurocortantes e medidas preventivas para hepatite $B$ adotadas por profissionais de Enfermagem nos serviços de urgência e emergência de Teresina, Piauí. Rev Bras Saúde Ocup. 2014; 39(130):175-83.

15. Martins AMEBL, Costa FM, Ferreira RC, Santos-Neto PE, Magalhães TA, Barbosa de Sá MA, et al. Factors associated with immunization against Hepatitis B among workers of the Family Health Strategy Program. Rev Bras Enferm. 2015; 68(1):84-92.

16. Pinto IC, Panobianco CSMM, Zacharias FCM, Bulgarelli AF, Carneiro TSG, Gomide MFS, et al. Analysis of job satisfaction of the nursing staff of a primary health care unit. Rev Gaúcha Enferm. 2014; 35(4):20-7.

17. Siqueira Julio R, Filardi MBS, Marziale MHP. Acidentes de trabalho com material biológico ocorridos em municípios de Minas Gerais. Rev Bras Enferm. 2014; 67(1):119-26.

18. Souza FO, Freitas PSP, Araújo TM, Gomes MR. Vacinação contra hepatite B e Anti-HBS entre trabalhadores da saúde. Cad Saúde Colet. 2015; 23(2):172-9.

19. Noronha DD, Vieira MRM, Vieira MM, Magalhães T A, Leite MTS. Acidentes ocupacionais ocorridos entre os profissionais de saúde do Hospital Universitário Clemente de Faria - HUCF. Motri. 2012; 8(2):67-77

20. Ministério da Saúde (Br). Lei 7.855 de 1989. Brasília (DF): Ministério da Saúde; 1989.

21. Ministério da Saúde (Br). Manual dos centros de referência para imunobiológicos especiais. Brasília (DF): Ministério da Saúde; 2006.

22. Ministério da Saúde ( $\mathrm{Br}$ ). Fundacentro. Manual de implementação: programa de prevenção de acidentes com materiais perfurocortantes em serviços de saúde. Brasília (DF): Ministério da Saúde; 2010.

23. Pinheiro J, Zeitoune RCG. Hepatite B: conhecimento e medidas de biossegurança e a saúde do trabalhador de enfermagem. Esc Anna Nery. 2008; 12(2):258-64.

24. Oliveira AC, Gonçalves JA. Acidente ocupacional por material perfurocortante entre profissionais de saúde de um centro cirúrgico. Rev Esc Enferm USP. 2010; 44(2):482-7.

25. Sanches GBS, Honer MR, Pontes ER, Aguiar JI, Ivo ML. Caracterização soroepidemiológica da infecção do vírus da hepatite $B$ em profissionais de saúde da atenção básica no Estado de Mato Grosso do Sul, Brasil. Rev Panam Infectol. 2008; 10(2):17-5.

26.Silva RJO, Athayde MJPM, Silva LGP, Braga EA, Giordano MV, Pedrosa ML. Vacinação anti-hepatite $B$ em profissionais da saúde. DST J Bras Doenças Sex Transm. 2003; 15(1):51-5.

27. Vos D, Götz HM, Richardus JH. Needlestick injury and accidental exposure to blood: the need for improving the hepatitis $B$ vaccination grade among health care workers outside the hospital. Am J Infect Control. 2006; 34(9):610-20.

28. Garcia LP, Facchini LA. Vacinação contra hepatite B entre trabalhadores da atenção básica à saúde. Cad Saude Publica. 2008; 24(5):1130-40.

29. Ministério da Saúde (Br). Portaria 597/GM 08 de Abril de 2004. Brasília (DF): Ministério da Saúde; 2004.

30. Yamazhan T, Durusoy R, Tasbakan MI, Tokem Y, Pullukcu H, Sipahi OR, et al. Turkish Nursing Hepatitis Study Group. Nursing students' immunisation status and knowledge about viral hepatitis 
in Turkey: a multi-centre cross-sectional study. Int Nurs Rev. 2011; 58(2):181-5.

31. Caetano JA, Soares E, Braquehais AR. Acidentes de trabalho com material biológico no cotidiano da enfermagem em unidade de alta complexidade. Enferm Glob. 2006; 1(9):1-12.

32. Moradi T, Maghaminejad F, Azizi FI. Quality of working life of nurses and its related factors. Nurs Midwifery Stud. 2014; 3(2):194-50.
33.Zimmerman RK, Raymund M, Janosky JE, Nowalk MP, Fine MJ. Sensitivity and specificity of patient self-report of influenza and pneumococcal polysaccharide vaccinations among elderly outpatients in diverse patient care strata. Vaccine. 2003; 21(13-14):1486-91.

34. Ministério da Saúde (Br). Secretaria de Gestão do Trabalho e da Educação na Saúde Secretaria de Vigilância em Saúde Mesa Nacional de Negociação Permanente do SUS. Protocolo $n^{\circ} 8$. Brasília (DF): Ministério da Saúde; 2011. 